\title{
Performance improvement of vehicular ad-hoc network with nature inspired biological computing algorithm
}

\author{
GHRCE, \\ Nagpur, 440016, India \\ Email: mehta.k15@gmail.com \\ Email: preetib123@yahoo.com \\ *Corresponding author
}

Komal Mehta* and Preeti Bajaj

\section{L.G. Malik}

Government College of Engineering (GCOEN),

Nagpur, 441108, India

Email: latesh.gagan@gmail.com

\begin{abstract}
VANET is a hybrid ad-hoc network between vehicles and road side units. Due to the high mobility of nodes, to find a stable data communication route in VANET is an open challenge. This paper introduces a new hybrid routing algorithm to find a stable route by using zone-based routing (ZBR), fuzzy logic, and NIBC algorithm. In proposed algorithm ZBR is used to divide the network into small and stable zones, fuzzy logic is used to find the quality of links between nodes, and NIBC to find the stable route in short time. Six techniques for the VANET has been implemented and compared in this paper. NS2.34 network simulator is used for simulations. Simulations were carried out for variable transmission rate and variable speed. Five performance parameters have been taken to analyse the results. Simulation results have shown that NIBC algorithms improve the performance of VANET.
\end{abstract}

Keywords: vehicular ad-hoc network; VANET; nature inspired biological computing; NIBC; artificial bee colony; $\mathrm{ABC}$; bacterial foraging optimisation; $\mathrm{BFO}$; particle swarm optimisation; PSO; performance parameters; performance improvement; fuzzy logic; AODV.

Reference to this paper should be made as follows: Mehta, K., Bajaj, P. and Malik, L.G. (2019) 'Performance improvement of vehicular ad-hoc network with nature inspired biological computing algorithm', Int. J. Hybrid Intelligence, Vol. 1, No. 1, pp.55-78.

Biographical notes: Komal Mehta is pursuing a $\mathrm{PhD}$ from the GH Raisoni College of Engineering, RTM Nagpur University, Nagpur, India. She has 14 years of experience and currently working as an Assistant Professor in the Bhagwan Parshuram Institute of Technology, Delhi. Her research interest includes vehicular ad hoc network, nature inspired biological computing (NIBC) and microwave engineering. She has more than 12 publications in refereed international conferences and journals. Her citations are 29 and h-index is 2 . 
Preeti Bajaj earned her Doctorate in Electronics Engineering in 2004. Having 25 years of experience, currently, she is the Director of the GHRCE, Nagpur. Her research interest includes intelligent transportation system, soft computing and hybrid intelligent systems. She is Fellow in the Institute of Engineers and IETE, a senior member in IEEE and an LM in ISTE and CSI. She is presently the Secretary in the IEEE India Council. She is also the Chair in IEEE Subsection, Nagpur. Many students pursued $\mathrm{PhD}$ and masters under her supervision. She has authored and co-authored 100 plus reviewed publications and three book chapters with citations 700 plus and h index 12 .

L.G. Malik completed her PhD in Computer Science and Engineering, in 2010. She is a Gold Medallist in BE and M.Tech. Having 18 years of experience, currently, she is an Associate Professor and the Head of the Department Computer Science and Engineering, Govt. College of Engineering, Nagpur. Her research interest includes document and pattern recognition, VANET and wireless networks, digital image processing, data mining and soft computing. She is fellow-IEEE member, LM-ISTE and CSI member. Eight students have submitted their $\mathrm{PhD}$ and many done masters under her supervision. She has authored and co-authored 100 plus reviewed publications and two book chapters with citations 800 plus and h index 13.

\section{Introduction}

Intelligent transportation system (ITS) is getting momentum in India specially to enhance the safety and improving the efficiency of overall movement of vehicle and traffic. Vehicular ad-hoc network (VANET) is an important component of ITS. Each vehicle in VANET, equipped with VANET devices, act as a node and responsible to provide communication through a wireless network via found route (http://www. internationaltransportforum.org). VANET consist a set of mobile nodes due to this network topology (physical connectivity of network) keep changing randomly so the topology of the wired network to find route cannot be applied directly to it. A variety of routing protocols for VANET has been proposed due to highly dynamic topology, limited resources at nodes and unavailability of centralised administration. VANET that consists of many community nodes is characterised by lack of stable connections. However, there are so many routing algorithms exists for providing connections between nodes to transmit data. These algorithms are broadly divided into five routing approaches; topology-based, position-based, geo-cast-based, zone-based and broadcast-based. Zone-based routing has been selected because it effectively handles the mobility issues of VANET and increases the stability. Zones are formed by grouping nodes with similar characteristics like same direction, same mobility to handle the dynamic nature of the network.

Dynamic nature of VANET can be handled by nature inspired biological computing (NIBC) also. Nature has variety of features to handle the various issues of VANET like diversity, dynamic, vigorous and captivating experience to resolve the complex problems (Binitha and Sathya, 2012). Potential research work has been done for VANET routing and found that the objectives of VANET can be achieved by NIBC techniques. Five NIBC algorithms have been designed and implemented: artificial bee, bacterial foraging optimisation (BFO), particle swarm optimisation (PSO), fuzzy BFO and fuzzy 
PSO in the zone-based routing for VANET. The performance of these is compared with AODV based on five performance parameters.

Performance parameters required the study of the characteristic behaviour of used protocols and technologies which gives the effectiveness of the network. Five performance parameters have been selected: packet delivery ratio (PDR), packet drop ratio, control overhead (CO), average delay and throughput to evaluate the performance of designed routing algorithms.

In the work, six algorithms for VANET routing has been presented and compared. The results have shown that NIBC-based algorithms improve the performance of VANET with respect to AODV which is not a NIBC algorithm. Simulation has also shown that using more than one intelligent approach performs better. Simulation carried out in NS2 for varying transmission rate and speed of nodes.

The organisation of the remaining paper is like that, the overview of related work done for VANET, in the field of routing, NIBC techniques and fuzzy logic is defined in Section 2. Section 3 consists of the flowchart of used methodology. Used NIBC algorithms explained in Section 4, with the help of flowchart. Simulation results and their comparison carried out in Section 5. Final conclusions defined in Section 6.

\section{Related works}

This section covers the literature survey and review of performance measure of VANET with and without nature inspired bio computing (NIBC). It discusses the outcomes of various research papers in the broad area of routing in VANET. Some of the reviews with critics are as follows.

Wang and Wang (2010) have developed cluster routing protocol which uses dubbed traffic infrastructure and handoff. It utilises the existing traffic infrastructures to form the cluster network. For improving packet delivery, only one hop range at the node was used. "The selection of dynamic cluster head for each vehicle that is based on handoff metric creates a time delay if the existing cluster head leaves from the cluster."

Shoaib and Song (2013) have proposed zone-based hierarchical routing protocol for reducing network overhead. They optimised the time interval to distribute network control packets by considering the mobility and traffic. "The PDR is good for the dense network but in the sparse network its performance degrades." In addition, if a single link changes among the nodes (although the links are stable) it will broadcast entire link state packet (LSP) in each zone to update routing table which in turn 'increases the CO'.

Kathirvel and Loyd (2014) established a link reliability to 'reduce packet drop ratio'. The combination of VANET and UMTS is used for longer connectivity among nodes. Cluster-based topology was used to reduce the complexity of the network but overhead increased. When real-time packet loss estimation is used to select the gateway or cluster head, results showed an "increase in PDR and decrease in packet drop ratio."

Patel and Jhaveri (2015) proposed a routing protocol. They used ACO and forward data packet only to the reliable nodes. They handle the packet drop attack and "improve the performance of VANET in terms of PDR, EED and CO."

Kaur and Kahlon (20150 proposed a hybrid protocol where they integrate the PSO with a HyBR algorithm for improving the routing efficiency of the HyBR algorithm. The 
results show that improvement in terms of delay and overhead but the PDR is almost same to HyBR.

Bitam and Mellouk (2011) proposed a routing protocol QoSBeeVANET (QBV). They used artificial bee algorithm to provide QoS in VANET. QBV is a multipath routing protocol which provides quality of service in VANET. "QBV outperforms DSDV and AODV in terms of PDR, EED and overhead, for urban settings."

Toutouh et al. (2011) configured vehicular data transfer protocol (VDTP) with five different NIBC algorithms. Results of experiments done for five different sizes of files and "showed that NIBC outperforms expert configured VDTP."

$\mathrm{Wu}$ et al. (2013) designed PFQ-AODV protocol for point to point communication which checks whether the wireless link is good or bad. This is done by considering mainly three metrics; availability of bandwidth (BW), quality of link and velocity of vehicle. They used Q-learning protocol and fuzzy logic in AODV. PFQ_AODV "provides improved PDR and EED in comparison to AODV, QLAODV and AODV-L. But, as the nodes increases or the speed increases, the delay also increases due to $\mathrm{CO}$ packets."

Jing et al. (2008) proposed a method to update route. Fuzzy logic is used to control the speed of sending packets. It considers network state parameters and QoS parameters for this. The study of the authors does not consider the route stability. The protocol is 'only suitable for low speed scenario'.

Hassnawi et al. (2014) investigated and analysed the effect of different packet size and rates on the VANET. They used AODV routing protocol. Results showed that at high mobility packet size should be small, which reduces the packet loss. When packet rate increases the packet drop ratio also increases for highway scenario.

Al-Nahari and Mohamad (2016) proposed RB-AOMDV routing protocol for MANET to decrease delay in rerouting. Simulation results have shown that at different packet rate of packet RB-AOMDV "improves PDR and average delay at the cost of normalised routing load."

Lee and Jeon (20150 proposed a multipath routing protocol for MANET. To handle the changing environment, biological attractor scheme is used in MMQR. Biological attractor scheme has improved the PDR, average delay for a dense network. However, "in sparse area, PDR of plain MMQR is greater than proposed one."

$\mathrm{Wu}$ et al. (2016) proposed a routing protocol based on a context-aware clustering approach. Proposed protocol also uses reinforcement learning-based approach to improve the efficiency of the route and shown improvement in PDR and average delay for both unicast and broadcast applications. But the throughput rapidly decreases when the distance between source and destination increased from $200 \mathrm{~m}$.

Elhoseny et al. (2017a, 2017b) proposed many GA-based algorithms to improve the performance of wireless sensors network (WSN). The proposed genetic algorithm-based self-organising network clustering method to optimise WSN cluster dynamically which increased the network lifetime (Yuan et al., 2016). In another paper, Elhoseny et al. (2017a) used a genetic algorithm to find cluster header $\mathrm{CH}$ dynamically based on six features. To improve network lifetime, Elhoseny et al. (2017b) proposed a model to find optimum sensor covers based on a number of factors. 


\section{Methodology}

Figure 1 has shown the adopted methodology for the proposed work. Zone-based routing approach has been selected as found from literature review that zone-based routing is more appropriate to handle the mobility and topology change of VANET. Selected of five performance parameters has been done as mentioned earlier. Setting some simulation parameters for carried out simulations in NS2 are shown in Table 1.

Figure 1 Adopted methodology

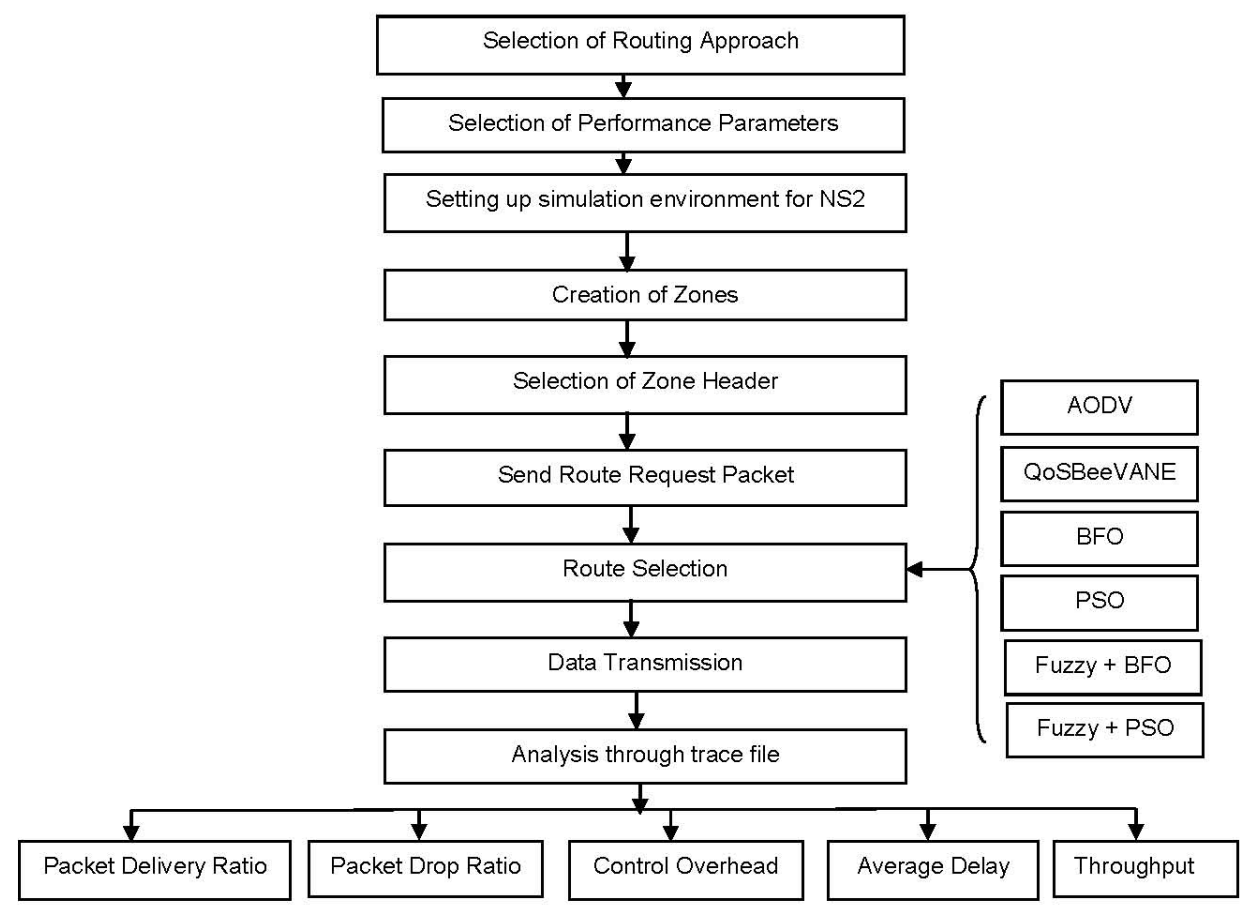

Table 1 Simulation settings and parameters

\begin{tabular}{lc}
\hline Number of nodes & 72 \\
Area size & $2,500 \times 2,500$ \\
Mac & IEEE 802.11 \\
Transmission range & $250 \mathrm{~m}$ \\
Simulation time & $20 \mathrm{sec}$ \\
Traffic source & $\mathrm{CBR}$ \\
Packet size & 512 bytes \\
Rate & Variable \\
Speed & Variable \\
\hline
\end{tabular}


Creation of zones and selection of zone head is done as shown in Figure 2. Here $v_{i}$ and $v_{j}$ are velocities of current node $i$ and neighbour node $j . E_{i}$ is energy of current node. $X_{i}$ is position of current node and $C_{Y}$ is the centre of zone $Y . C_{Y}$ is zone head of zone $Y$, initially the current node itself is zone head laterally zone head update according to the fitness function $\left(F_{i}\right)$.

Figure 2 Creation of zone and selection of zone-head

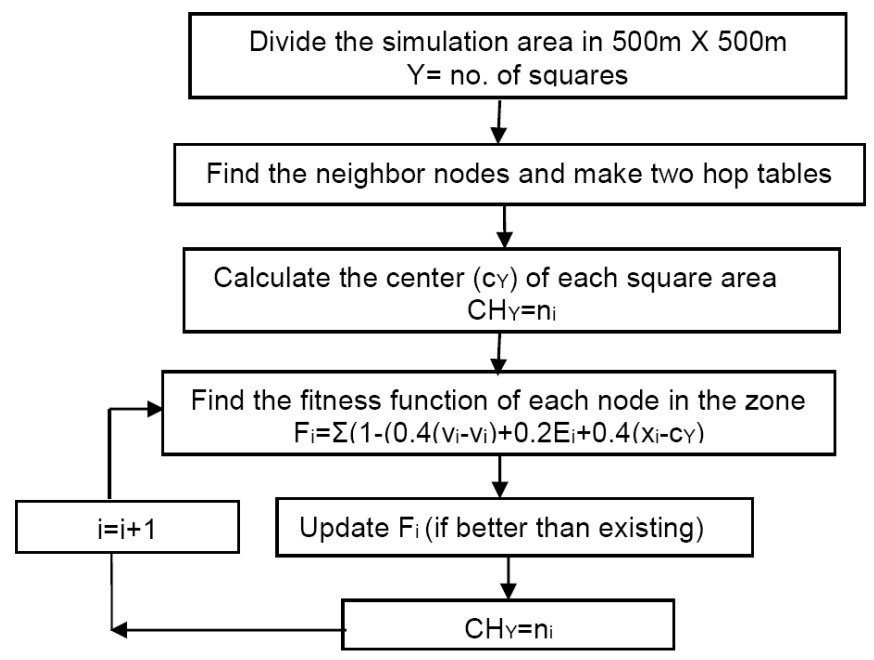

When any node wants to send data it initiate for route through which data can be transmitted. Source node generate route request message for this RREQ.

Figure 3 RREQ message received by node 39 from node 53 (see online version for colours)

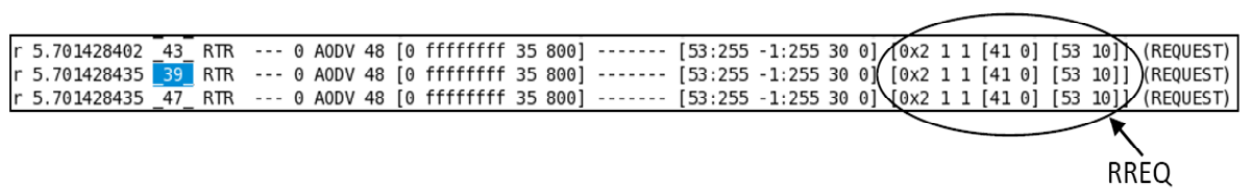

Figure 3 shows RREQ message received (i.e., $r$ ) by node 39 at time 5.701428435 second. It is a routing (RTR) request. The unique ID of packet is 0 and size is 48 byte. Its payload type is AODV. [0 ffffffff 35800 ] these are MAC layer details regarding MAC source and destination address with delay at MAC layer. Then [53:255 -1:255 30 0] indicate IP source and destination addresses and port is 255 for both. The time to live is 30 hops. $0 \times 2$ indicate the tagged ID of RREQ packet. Hop count and broadcast ID both are 1. [41 0 ] and [53 10] is IP address and sequence number of destination and source, respectively.

Route selection done by all the six implemented routing algorithms: AODV, QBV, BFO, PSO, fuzzy BFO and fuzzy PSO. They all found the route according to their expertise, as explained in Section 4. After finding the route, source node start sending data through that found route. When the simulation complete, analysed the trace files and evaluated the performance parameters. 


\section{Flowcharts of AODV and used NIBCs}

\subsection{AODV with zone-based routing}

First step of Figure 4 is creation of zone, which is done as explained in Section 3. When any node wants to send data it first check that is destination node is in its two hop vicinity, if yes then it directly sends the data to it but if not then it sends RREQ to its zone head $(\mathrm{CH})$. Now, zone head will check is destination node is in its own zone, if yes then send the RREQ to it or if not then $\mathrm{CH}$ forward the RREQ nearby CHs. Like this, each zone head first search their own zone for destination node and if not found then forward RREQ to nearby zone heads until the RREQ reached to destination node. The destination node sends RREP to source node through the found route, after receiving the RREQ packet (Wu et al., 2013). When source node received the RREP packet, it starts transmitting the data to destination node through the found route. After the simulation over trace file has been analysed and evaluate the values of all the five performance parameters.

Figure 4 Flowchart of AODV with zone-based routing

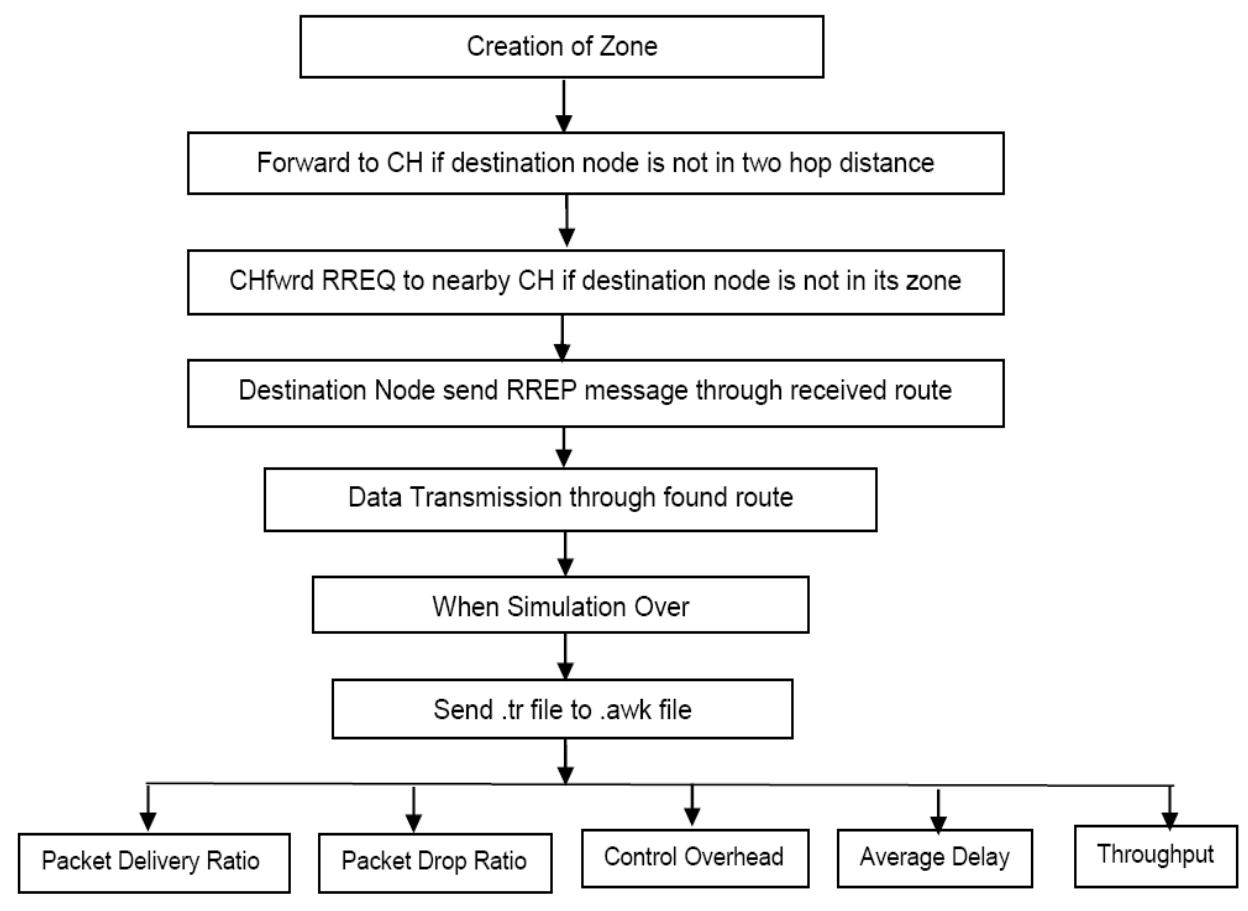

\subsection{QBV with zone-based routing}

The first phase of the flowchart shown in Figure 5 is common for all the designed algorithms and it explained in Section 3. 
Figure 5 Flowchart of QBV with zone-based routing

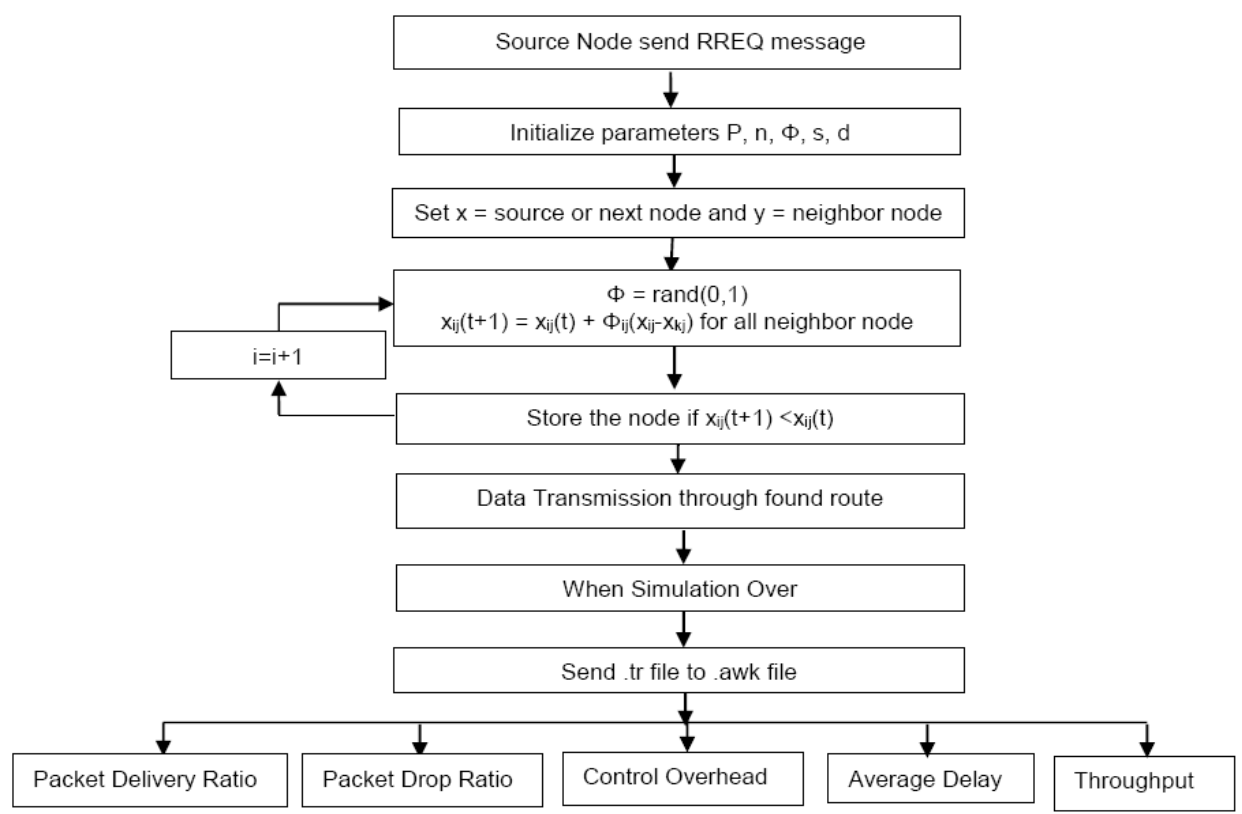

QBV is inspired by communication in swarms of bees. It is a reactive, distributed and topology-based multipath routing protocol (Bitam and Mellouk, 2014).

Initialisation of some parameters for defined algorithm is:

$P \quad$ search space

$\Phi \operatorname{rand}(0,1)$

$s \quad$ source node

$d$ destination node

$n$ total no. of nodes.

Assumed that all the nodes are GPS enabled and there is no curve on the road. QBV used the relative positions of the nodes to find the route. Equation (1) is used to find the next node. Here, $k$ is neighbour node of node $j$.

$$
x_{i j}(t+1)=x_{i j}(t)+\Phi_{i j}\left(x_{i j}-x_{k j}\right)
$$

here $\Phi=\operatorname{rand}(0,1)$, by default the value of $\Phi=1$, it only changes randomly when node stuck in a loop of local best. $x_{i j}$ is the position difference between current node $i$ and neighbour $j$, means

$$
x_{i j}=\left[\left(x_{i}-x_{j}\right),\left(y_{i}-y_{j}\right)\right]
$$




\subsection{Bacterial foraging optimised zone-based routing}

Flow chart of bacterial foraging optimised zone-based routing (BFOZBR) is shown in Figure 6. Inputs to BFOZBR algorithm are (Kalambe et al., 2015):

$c$ total number of zone head

$P \quad$ number of nodes

$e \quad c$ because only sorting the nodes not deleting

$M_{c} \quad$ twice the chemotactic steps.

Figure 6 Flowchart of BFOZBR

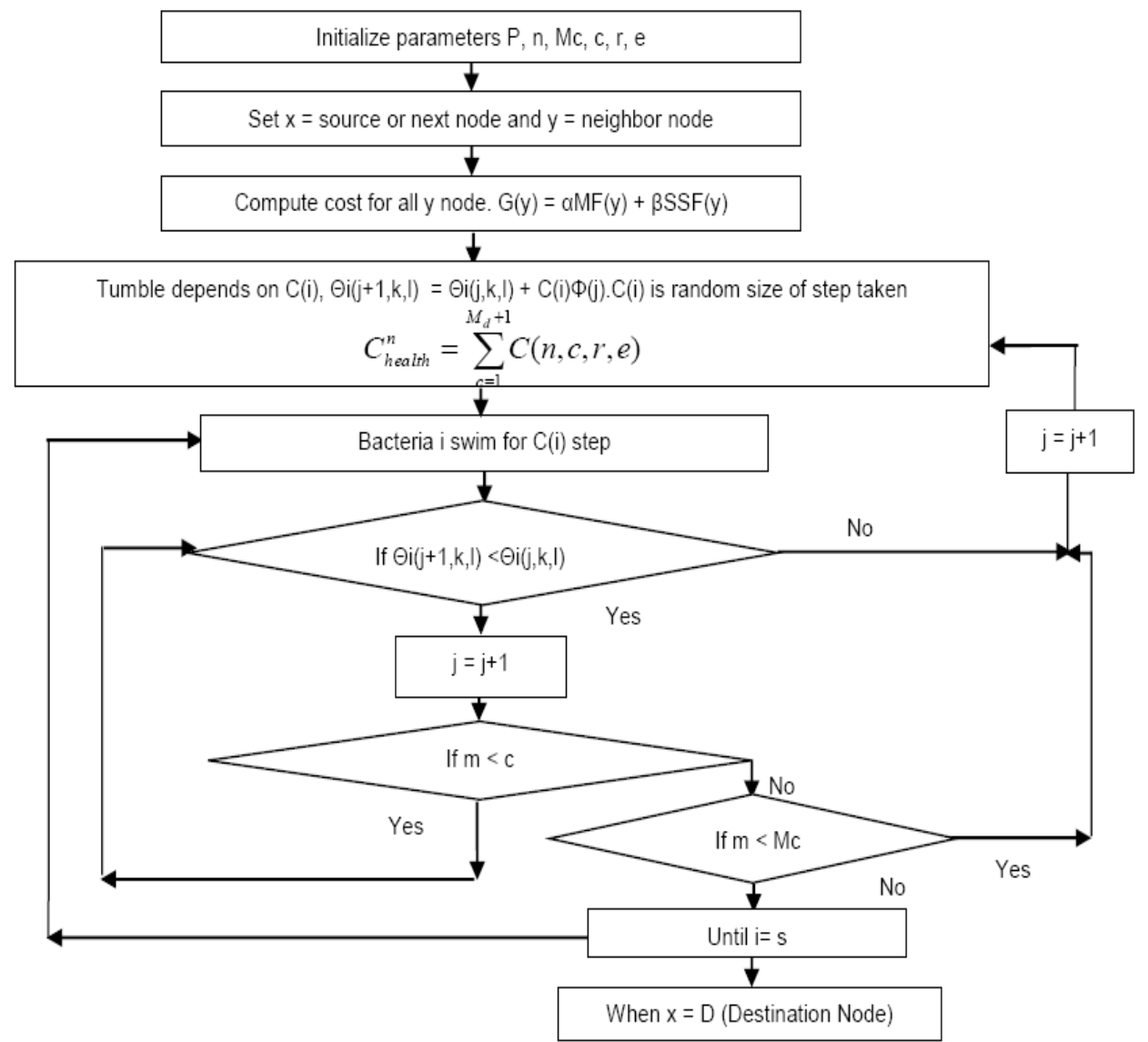

In the proposed routing protocol, two merit factors were selected to evaluate the link status (LS). Values of these two merit factors were calculated to provide the cost of each node (Wu et al., 2012). 
- Mobility factor $(M F)$ : it indicates the relative motion of current node and neighbour node and calculated by formula (3):

$$
M F(y)=\left(1-\frac{\left|d_{t}(y)-d_{t-1}(y)\right|}{R}\right)
$$

Here, $d_{t}(y)$ and $d_{t-1}(y)$ is the distance between current node and neighbour node at time $t$ and $t-1$, respectively. $R$ is transmission range. Low relative movement gives large MF. $y$ is a neighbour node. MF initialised to 0 .

- Signal strength factor: it indicates the signal strength of neighbour node and calculated by formula (4):

$$
\operatorname{SSF}(y)=\left(1-\frac{R X_{\text {Threshold }}}{R X_{\text {Power }}}\right)
$$

Here, $R X_{\text {Threshold }}$ is reception threshold power and $R X_{\text {Power }}$ is received the signal power of neighbour node $y$. SSF initialised to 0 . High received power gives high signal strength factor.

Cost of a node calculated by equation (5)

$$
G(y)=\alpha M F(y)+\beta S S F(y)
$$

Here, $M F(y)$ is MF of neighbour node $y$ and $S S F$ is the signal strength of $y$. The value of $\alpha$ and $\beta$ is 0.5 and the standard values of $\alpha$ and $\beta$ has been taken.

To calculate the cost function, assumed that all the nodes are GPS enabled and there is no curve in the road. The value of reception threshold power $R X_{\text {Threshold }}$ is $4.4613 \mathrm{e}-10$. We have taken $250 \mathrm{~m}$ transmission range for simulation.

The selection of the next hop node is done by finding the health (cost) of the bacterium like: for each $\mathrm{n}=1,2, \ldots \ldots, P$, let (Das et al., 2009)

$$
C_{\text {health }}^{n}=\sum_{c=1}^{M_{d}+1} C(n, c, r, e)
$$

The node having highest cost value gets dispersed.

If any node continuously not dispersing any neighbour node, than there are chances that the node gets stuck in local best solution. To handle this situation, tumbling take place.

Set elimination step $e=3$. Means, if a node for three-times continuously do not disperse any node than multiply its neighbour list with a random number (tumbling). Equation 7 changes the sequence of the neighbours. Where $\Phi(j)$ is the selected neighbour node.

$$
\Phi i(j+1, k, l)=\Phi i(j, k, l)+C(i) \Phi(j)
$$

\subsection{Particle swarm optimised zone-based routing}

First, find out the position difference between source node and destination node $\left(\left(x_{s}-x_{D}\right)\right.$, $\left.\left(y_{s}-y_{D}\right)\right)$. Then, calculate two merit factors as shown in Figure 7. 
Figure 7 Flowchart of particle swarm optimised zone-based routing (PSOZBR)

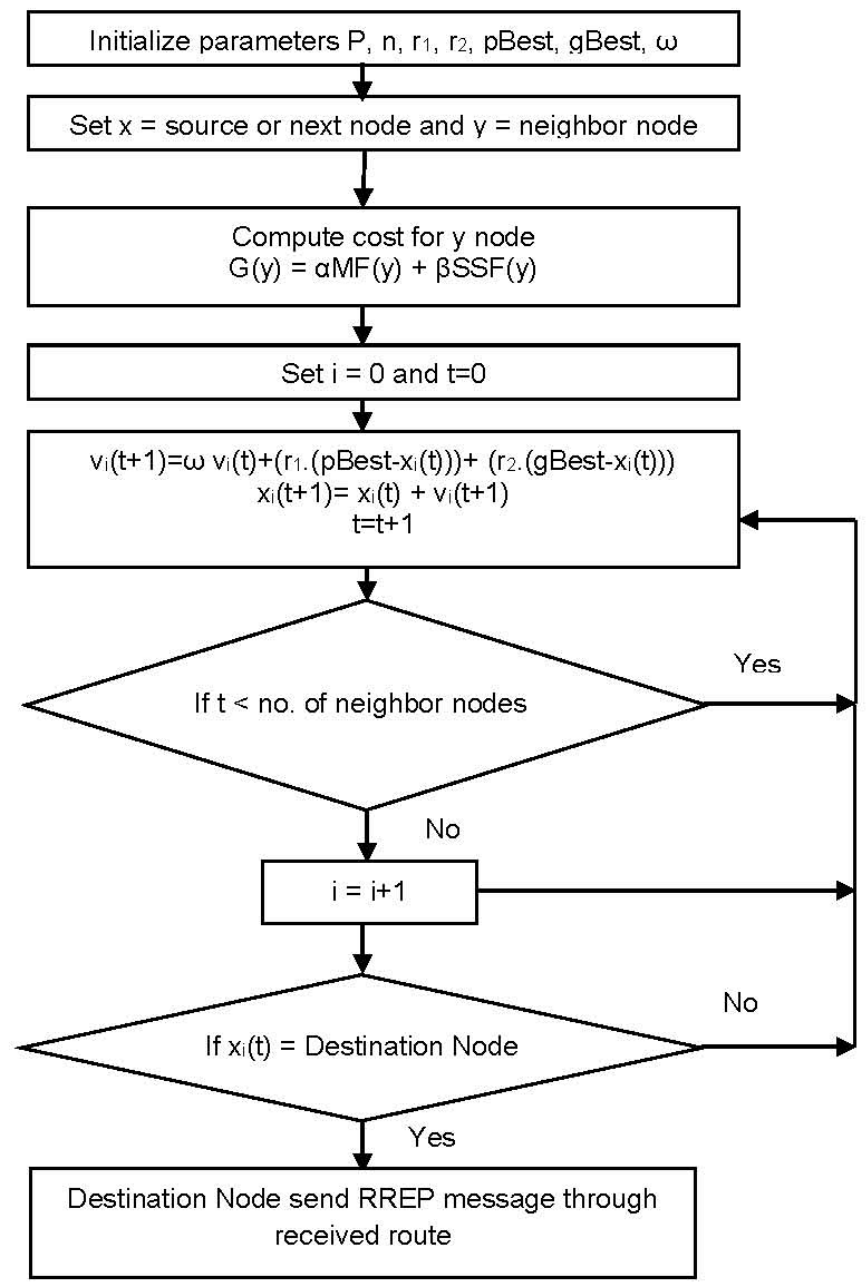

Now, calculate $v_{i}(t+1)$ based on the cost value $G(y)$ as calculated above in BFOZBR.

$$
v_{i}(t+1)=\omega v_{i}(t)+\left(r_{1} \cdot\left(p B e s t-x_{i}(t)\right)\right)+\left(r_{2} \cdot\left(g B e s t-x_{i}(t)\right)\right)
$$

Not modifying the PSO, so $\omega=1$ and $r_{1}$ and $r_{2}$ are $\operatorname{rand}(0,1)$ (Kalambe et al., 2015). The value of $r_{1}$ and $r_{2}=0.5$ are taken as a standard value. The value of $r_{1}$ and $r_{2}$ changes randomly if continuously getting same node as a next node. Initially, the value of $p B e s t$ and gBest are of source node itself. Then, select the nodes according to their position difference with forwarding node.

Calculate $v_{i}(t+1)$ for all selected nodes and select the best one to update the position $x_{i}(t+1)=x_{i}(t)$ and best $v_{i}(t+1)$ become $g$ Best. And node's own cost becomes pBest. 


\subsection{Fuzzy bacterial foraging optimised zone-based routing}

Fuzzy logic has been added to determine the cost function to improve the performance of VANET. Three input merit factors are used to judge the LS more preciously, these are:

- BW factor: it depends on the channel ideal ratio $(C I R)$ which is a ratio of the period when the channel was idle and total period.

$$
C I R=\frac{\text { idle time period of channel }}{\text { total sensing time }}
$$

The final value of $C I R$ calculated by averaging the value of $C I R$ at $t-1$ and $t$ time. This information transmitted in hello and RREQ packets by each node to calculate the $\mathrm{BW}$ factor using equation (10):

$$
B W F=\min (\operatorname{CIR}(n), C I R(y))
$$

Here, $n$ is current node and $y$ is next node.

- $M F$ : it depends on the neighbour information, so GPS not required for getting information on the position of the nodes. For this reason, additional coverage of node $y$ inside a zone, i.e., $A C(n, y)$ is used to represent sets of nodes that are one-hop neighbours of the node y but not of current node $n . A C(n, y)$ described as equation (11):

$$
A C(n, y)=\overline{O(n)} \cap O(y) \quad \text { where } y \in O(n)
$$

where $O(y)$ and $O(n)$ represent set of a one-hop neighbour of node $y$ and $n$, respectively. On receiving the hello message from current node, the MF calculated for sender node using equation (12):

$$
M F_{t}(n, y)= \begin{cases}\sqrt{\frac{\left|A C_{t}(n, y) \cap A C_{t-1}(n, y)\right|}{\left|A C_{t}(n, y) \cup A C_{t-1}(n, y)\right|}, A C_{t}(n, y) \cup A C_{t-1}(n, y) \neq \varnothing} \\ 0, \quad \text { otherwise }\end{cases}
$$

where $A C_{t}(n, y)$ and $A C_{t-1}(n, y)$ represent the present value and the previous value, respectively. By averaging the value of MF at $t-1$ and $t$ time, the relative movement of nodes has been considered.

- Link quality factor (LQF): it depends on the hello reception ratio (HRR). Each node calculates reception ratio and updates it in the LSPs database. Hello messages sent with a predefined time interval, $1 \mathrm{~s}$. HRR that depends on the number of received hello messages in the last $10 \mathrm{~s}$ reduces the effect of packet collision event. HRR calculated as given in equation (13):

$$
\operatorname{HRR}_{t}(n, y)=\left(\begin{array}{ll}
\frac{N U M_{r}(n, y)}{N U M_{s}(y)}, & N U M_{s}(y) \geq 10 \\
\frac{N U M_{r}(n, y)}{N U M_{s}(y)} \cdot\left(1-\left(\frac{1}{2}\right)^{N U M_{s}(y)}\right), & \text { otherwise }
\end{array}\right.
$$


where $\operatorname{NUM}_{r}(n, y)$ represents the number of hello messages received at $n$ from the node $y, N U M_{s}(y)$ represents a number of hello messages sent from node $y$.

Then, the LQF calculated as equation (14):

$$
L Q F(n, y) \leftarrow(1-\alpha) L Q F_{t-1}(n, y)+\alpha \times \operatorname{HRR}_{t}(n, y)
$$

here, $\alpha=0.75$ it is a standard value.

$L Q F_{t-1}(y)$ initialised to 0 . After calculation of the $L Q F, L Q F_{t-1}(n, y)$ updated as:

$$
L Q F_{t-1}(n, y)= \begin{cases}0 & N U M_{s}(y)<10 \\ L Q F(n, y) & \text { otherwise }\end{cases}
$$

Now, after finding the values of these merit factors fuzzification take place which converts these crisp values in fuzzy values using membership functions as defined in Figures 9, 10 and 11. Figure 8 shows the flowchart of BFOZBR.

Some set of rules defined based on fuzzy input values (BW, MF and LQF). In rule evaluation, analyse these rules and finds out a fuzzy output value. Here, LS is an output variable. Table 2 shows some fuzzy rules for different combinations of BW, MF, LQF and fuzzy value of output LS.

Table 2 Table of rules

\begin{tabular}{lcccc}
\hline Rule no. & $B W$ & $M F$ & $L Q$ & $L S$ \\
\hline 1 & Large & Slow & Good & Perfect \\
2 & Large & Slow & Medium & Good \\
3 & Large & Medium & Medium & Acceptable \\
4 & Large & Slow & Bad & Unpreferable \\
5 & Large & Fast & Medium & Bad \\
6 & Small & Medium & Bad & Very Bad \\
7 & Large & Medium & Good & Good \\
8 & Medium & Medium & Medium & Unpreferable \\
9 & Medium & Medium & Good & Acceptable \\
10 & Large & Fast & Good & Unpreferable \\
\hline
\end{tabular}

Here, multiple rules applied at the same time, so to avoid the ambiguity, min-max method has been selected. The minimum value selected for, each rule to find out LS. When more than one, same consequent comes, than the maximum value of consequent, selected to find out LS. Here, the values of BW, MF and LQ are as follows:

- $\quad \mathrm{BW}$ is $\{$ large $=1$, medium $=0$, small $=0\}$

- $\quad \mathrm{MF}$ is $\{$ slow $=0.7$, medium $=0.3$, fast $=0\}$

- $\mathrm{LQF}$ is $\{\operatorname{good}=0.6$, medium $=0.4, \mathrm{bad}=0\}$.

Now, this fuzzy output LS converted into crisp output using defuzzification. Centre of gravity method is used for defuzzification. Figure 12 shows membership function of output. 
Figure 8 Flowchart of fuzzy bacterial foraging optimised zone-based routing (FBFOZBR)

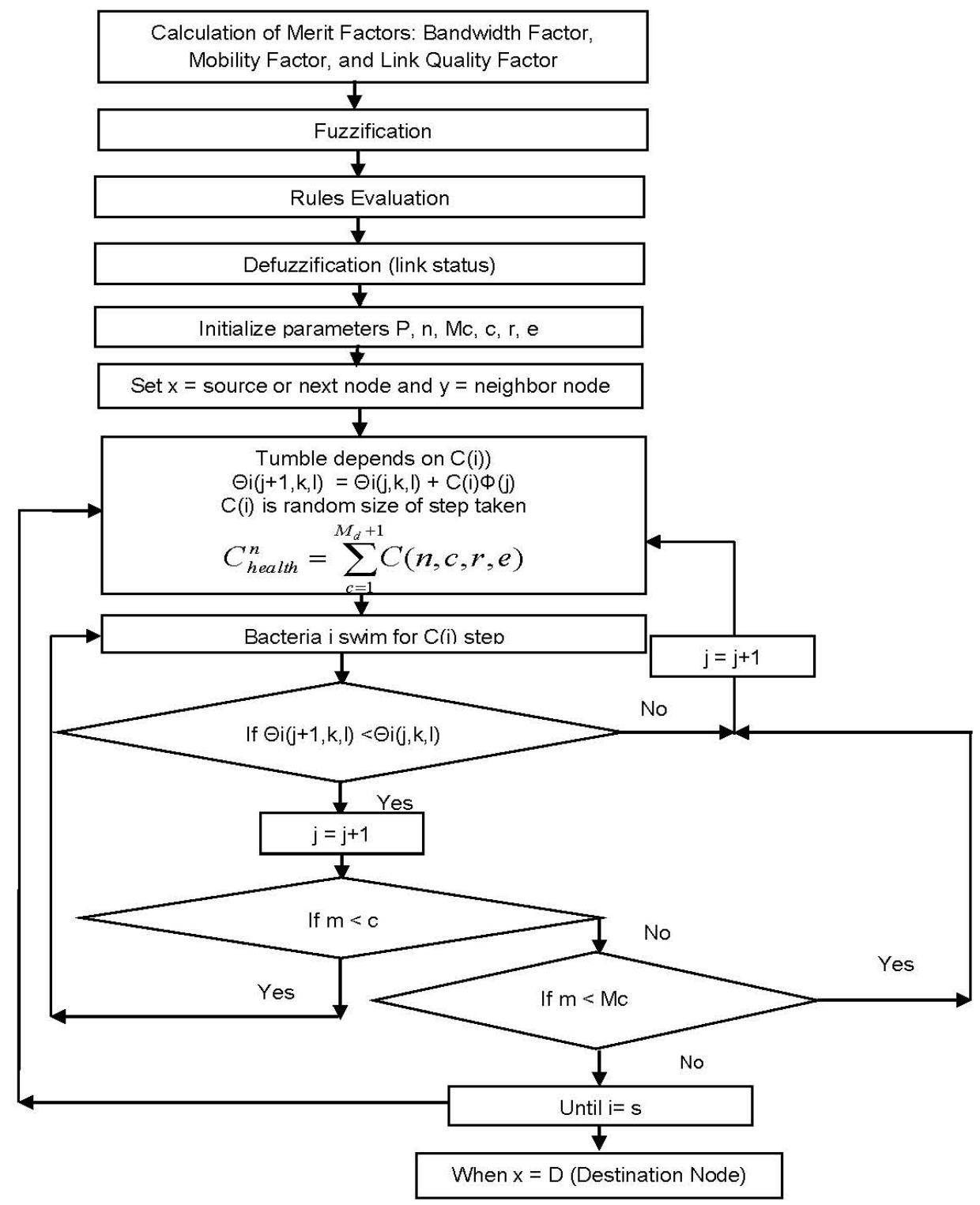

Formula (15) describes the defuzzifier technique.

$$
\text { Fuzzy_cost }=\left[\sum_{\text {all rules }} k_{i} * \eta\left(k_{i}\right)\right] /\left[\sum_{\text {all rules }} \eta\left(k_{i}\right)\right]
$$

Fuzzy_cost represent crisp output (LS), $k_{i}$ represent all fuzzy variables and $\eta\left(k_{i}\right)$ is fuzzy output $(i)$.

Check the cost of each neighbour node and dispersed the node having lowest cost value using equation (6). BFO has been used as explained in BFOZBR after finding the LS. Tumbling has been done to get out from local minima. 
Figure 9 Membership function of BW

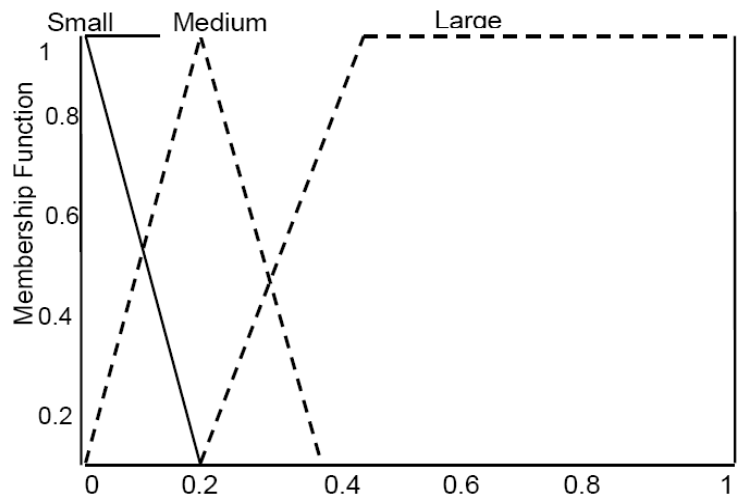

Figure 10 Membership function of BW

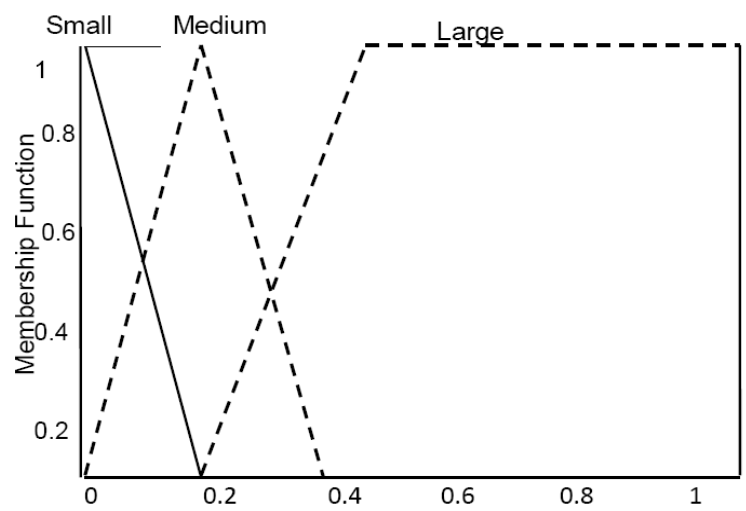

Figure 11 Membership function of link quality

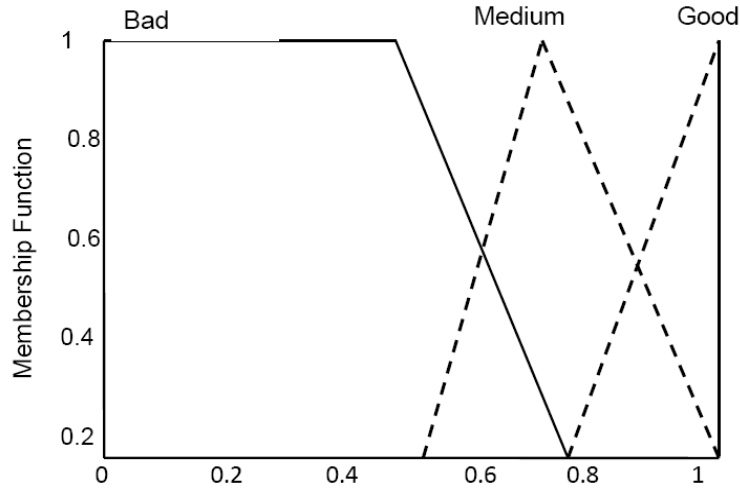


Figure 12 Output membership function

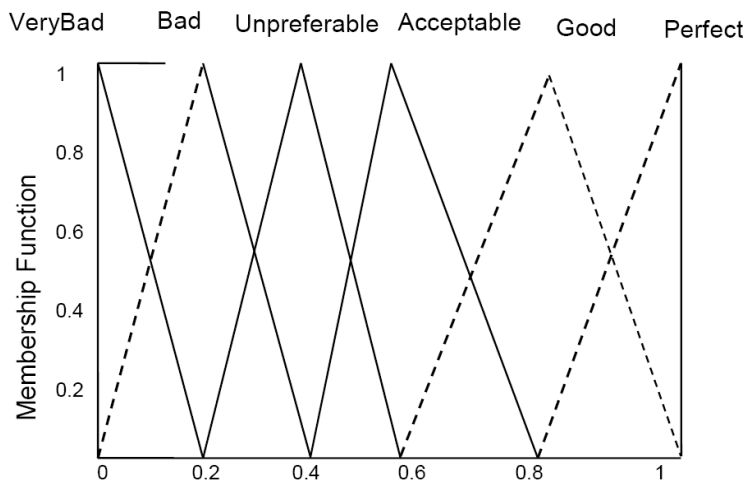

\subsection{Fuzzy particle swarm optimised zone-based routing}

Figure 13 shows the flowchart of fuzzy particle swarm optimised zone-based routing (FPSOZBR). This finds out the LS of every link using fuzzy logic as explained in FBFOZBR.

Figure 13 Flowchart of FPSOZBR

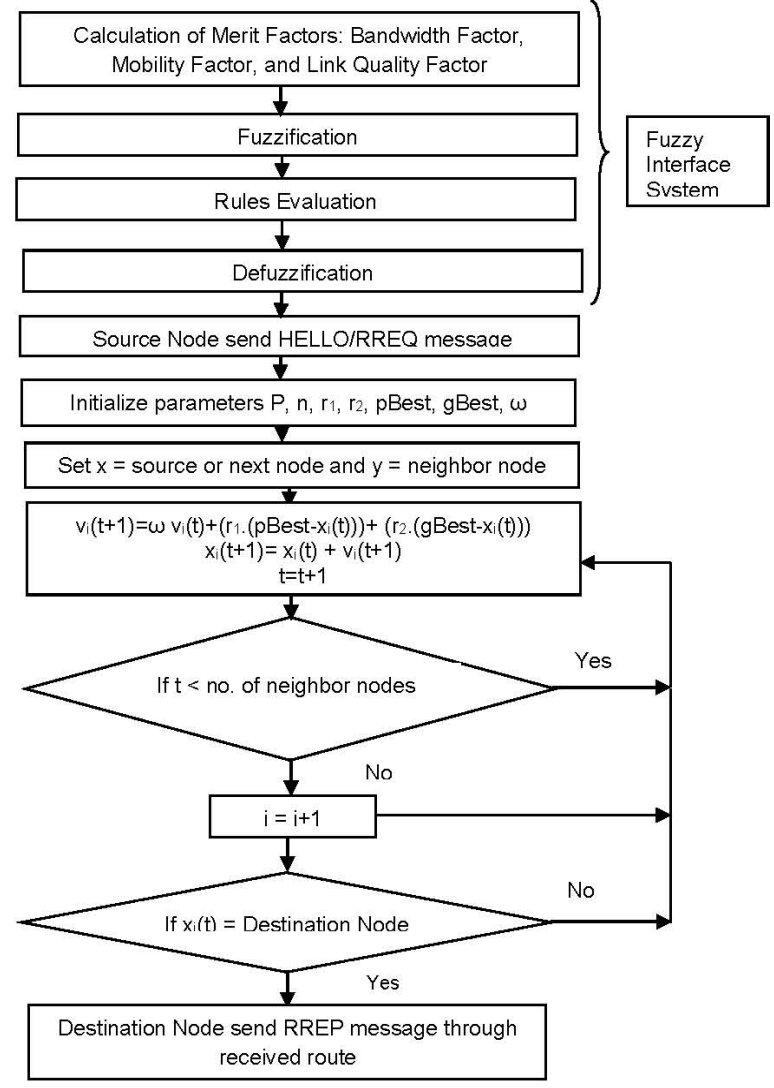


When any node wants to transmit data it will send the RREQ message and initialise the parameters $P, n, r_{1}, r_{2}, p B e s t, g B e s t$ and $\omega$ as explained in PSOZBR. Initially, the $p B e s t$ and gBest are same then it changes according to the selection of next forwarding node. Velocity and position $v_{i}$ and $x_{i}$, respectively, also updated with the selection of next forwarding node as mentioned in PSOZBR.

\section{Results}

Simulation are carried out for ten cases initially varied the transmission rate from $100 \mathrm{Kbps}$ to $500 \mathrm{Kbps}$ with the difference of $100 \mathrm{Kbps}$ at speed $5 \mathrm{~m} / \mathrm{s}$. then fixed the transmission rate at $100 \mathrm{Kbps}$ and changed the speed of nodes from $5 \mathrm{~m} / \mathrm{s}$ to $25 \mathrm{~m} / \mathrm{s}$ with the change of $5 \mathrm{~m} / \mathrm{s}$.

When simulation over, analysed the generated trace file and calculated the performance parameters. The comparison of designed algorithms is made based on performance parameters as follows.

\subsection{Packet delivery ratio}

Table 3 shows the comparison of all the designed algorithms for PDR improvement with respect to AODV. After analysing Table 3, it found that FBFOZBR has maximum PDR. $\mathrm{BFO}$ and FBFOZBR both have more PDR compare to AODV. As analysed from Table 3 that for most of the simulation combinations QBV, PSOZBR and FPSOZBR have more PDR compare to AODV. Also, observed that at $100 \mathrm{Kbps}$ transmission rate for variable speed FPSOZBR has more PDR compared to AODV.

\subsection{Packet drop ratio}

Table 4 shows the comparison of all the designed algorithms and performance improvement in terms of packet drop ratio with respect to AODV. Here, negative sign indicates low packet drop ratio, which shows improvement in performance. By analysing Table 4, it found that FBFOZBR has minimum packet drop ratio. QBV, BFOZBR and FBFOZBR all three have low packet drop ratio compare to AODV. FPSOZBR has highest performance for variable speed at $100 \mathrm{Kbps}$ transmission rate.

\subsection{Control overhead}

By analysing Table 5, we found that FPSOZBR has minimum CO. FBFOZBR has also less CO compare to AODV. QBV and PSOZBR have low CO at $100 \mathrm{Kbps}$ for variable speed. When used fuzzy logic in PSOZBR and BFOZBR CO decreased. 
72

K. Mehta et al.

Table 3 Comparison in terms of PDR

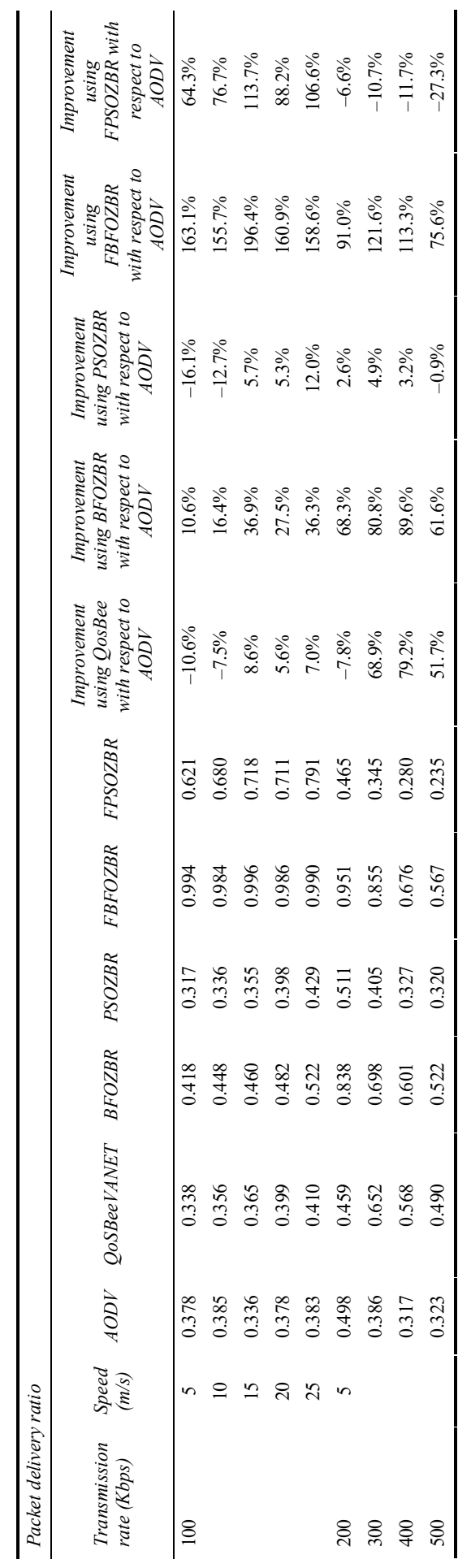


Table 4 Comparison in terms of packet drop ratio

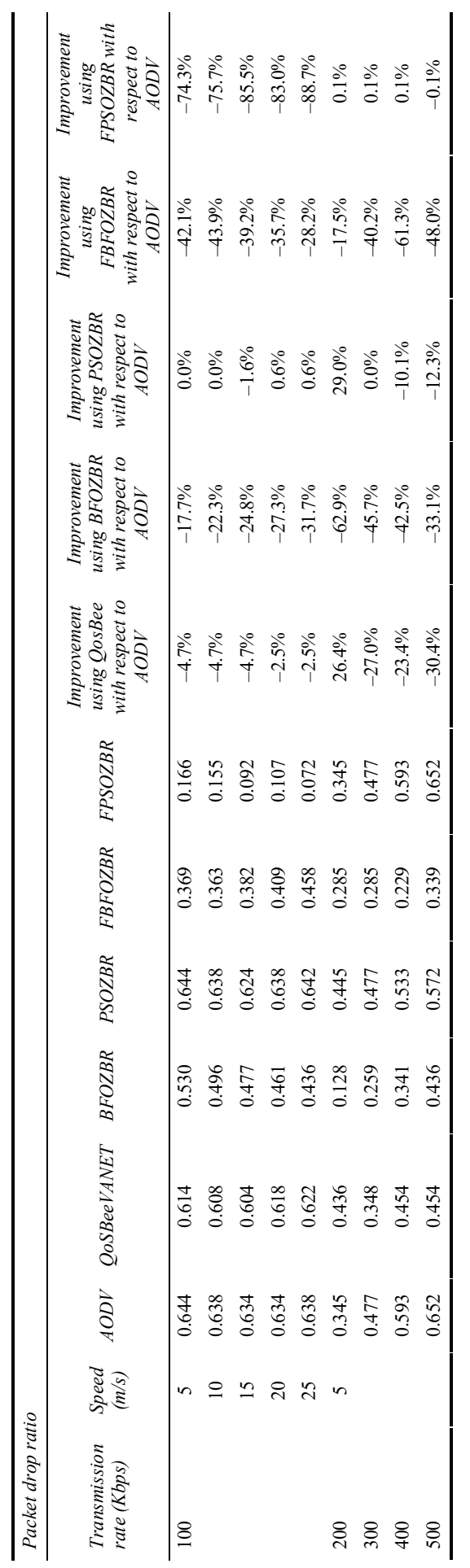


Table 5 Comparison in terms of $\mathrm{CO}$

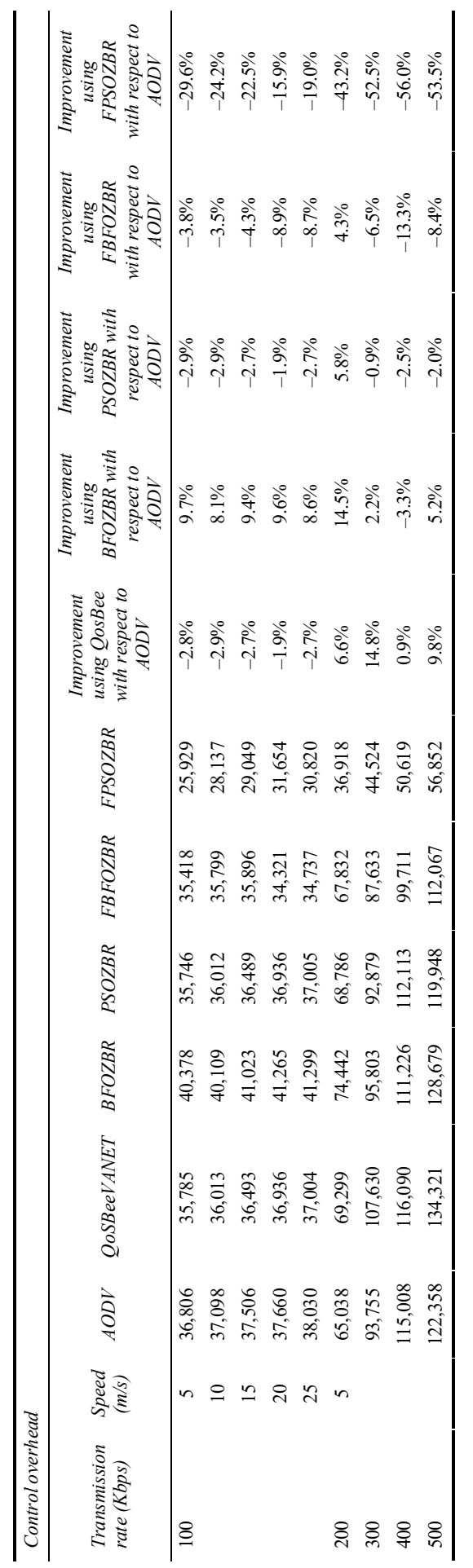


Table 6 Comparison in terms of average delay

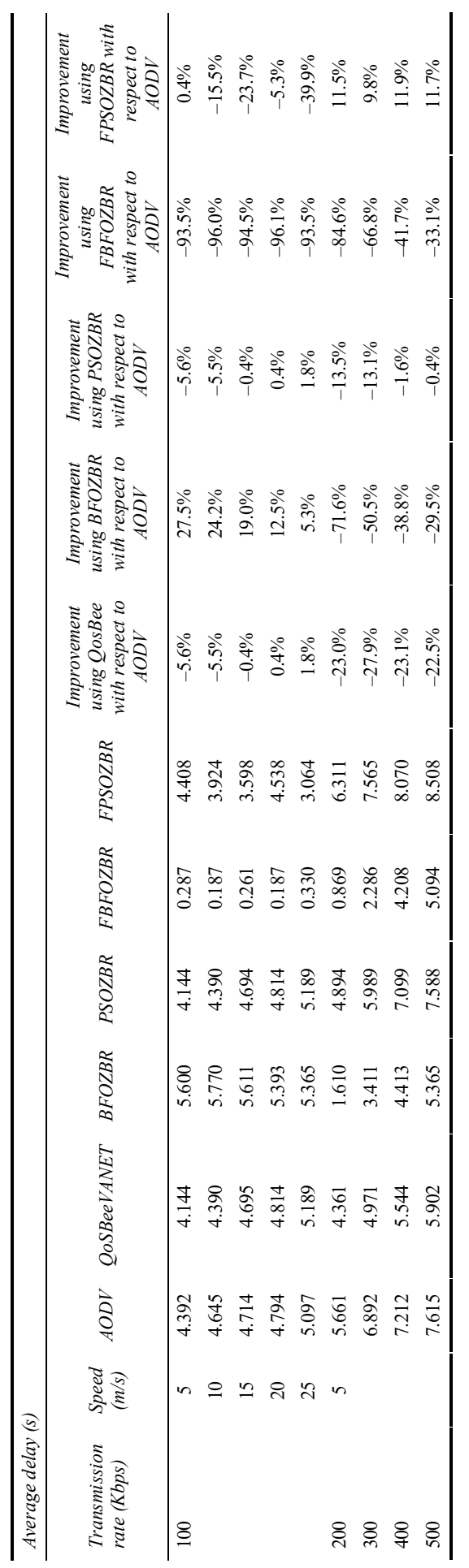


Table 7 Comparison in terms of throughput

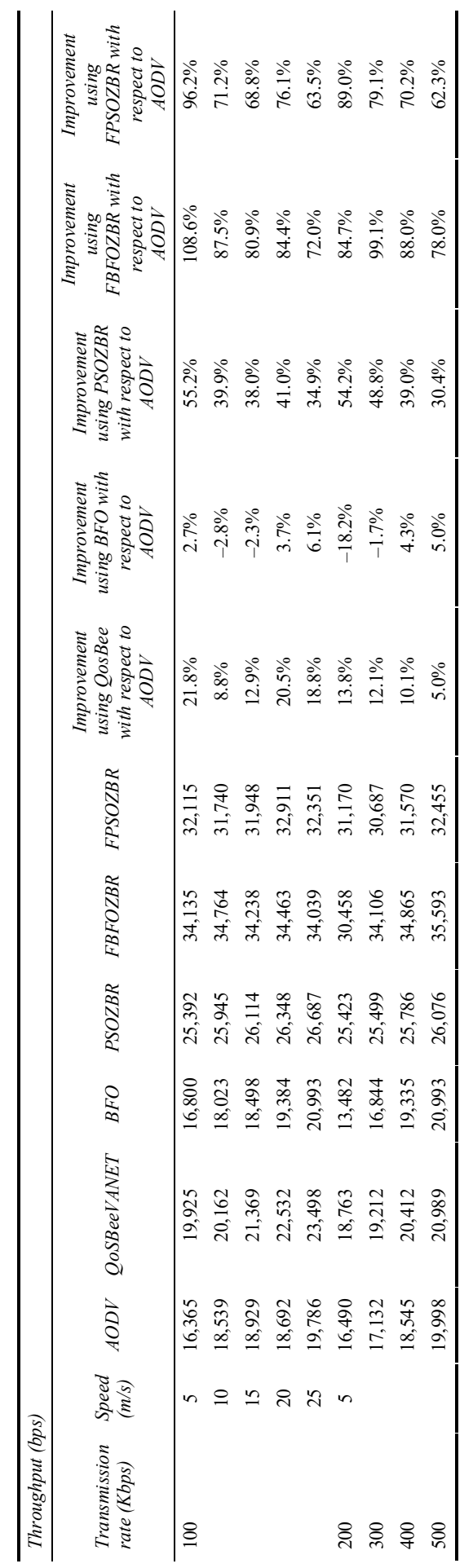




\subsection{Average delay}

Table 6 shows that FBFOZBR has a minimum average delay. QBV has either less or almost equal average delay to AODV.

\subsection{Throughput}

By analysing Table 7, we found that FBFOZBR has maximum throughput. All the designed NIBC algorithms except BFOZBR have high throughput compare to AODV. When used fuzzy logic in BFOZBR, throughput increased.

\section{Conclusions}

It has been concluded from the results that at varying transmission rate or varying speed, NIBC algorithms performed better than AODV. Although there are few combinations in which the performance of simple NIBC algorithms are not better but almost equal to AODV. It has been observed that hybrid NIBC algorithms outperformed over AODV for all the simulation combinations. Proposed algorithm FBFOZBR has shown improvement in average PDR by $92 \%$, average packet drop ratio by $39 \%$, CO by $7 \%$, average delay by $77 \%$ and throughput by $86 \%$, compared to AODV. It has been also observed from the results that at varying speed NIBC algorithms performed a more appropriate comparison to non-NIBC algorithms.

\section{References}

Al-Nahari, A. and Mohamad, M.M. (2016) 'Receiver-based ad hoc on demand multipath routing protocol for mobile ad hoc networks', PLoS ONE, June, Vol. 11, No. 6, e0156670, DOI: 10.1371/journal pone. 0156670 .

Binitha, S. and Sathya, S.S (2012) 'A survey of bio inspired optimization algorithms', International Journal of Soft Computing and Engineering (IJSCE), May, Vol. 2, No. 2, pp.137-151, ISSN: 2231-2307.

Bitam, S. and Mellouk, A. (2011) 'QoS swarm bee routing protocol for vehicular ad hoc networks', IEEE International Conference on Communications, pp.1-5.

Bitam, S. and Mellouk, A. (2014) Bio-Inspired Routing Protocols for Vehicular Ad-Hoc Networks, Technology and Engineering, John Wiley \& Sons, DOI:10.1002/9781119004967.

Das, S., Biswas, A., Dasgupta, S. and Abraham, A. (2009) 'Bacterial foraging optimization algorithm: theoretical foundations, analysis, and applications', Foundations of Computational Intelligence, Vol. 3, pp.23-55, Springer, Berlin Heidelberg.

Elhoseny, M., Farouk, A., Zhou, N., Wang, M-M., Abdalla, S. and Batle, J. (2017a) 'Dynamic multi-hop clustering in a wireless sensor network: performance improvement', Wireless Pers. Communication, March, pp.3733-3753, Springer, DOI: 10.1007/s11277-017-4023-8.

Elhoseny, M., Tharwat, A., Farouk, A. and Hassanien, A.E. (2017b) 'K-coverage model based on genetic algorithm to extend WSN lifetime', IEEE Sensors Letters, August, Vol. 1, No. 4, pp.1-4.

Hassnawi, L.A. et al. (2014) 'Measurement study on packet size and packet rate effects over vehicular ad hoc network performance', Journal of Theoretical and Applied Information Technology, Vol. 70, No. 3, pp.475-481. 
Jing, Z. et al. (2008) 'Service-aware multi-constrained routing protocol with QoS guarantee based on fuzzy logic', 22nd IEEE International Conference on Advanced Information Networking and Applications - Workshop, pp.763-767.

Kalambe, K.D., Deshmukh, A.R. and Dorle, S.S. (2015) 'Particle swarm optimization based routing protocol for vehicular ad hoc network', International Journal of Engineering Research and General Science, Vol. 3, No. 1, pp.1375-1382.

Karaboga, D. (2010) ‘Artificial bee colony algorithm', Scholarpedia, Vol. 5, No. 3, p.6915.

Kathirvel, C. and Loyd, D. (2014) 'Optimized hybrid wireless mesh protocol using estimation of packet loss rate algorithm for VANET', International Journal of Computer Science and Mobile Applications, Vol. 2, No. 3, pp.117-123.

Kaur, A. and Kahlon, N.K. (2015) 'Swarm based enhanced hybrid routing protocol in VANETs', International Journal of Advanced Research in Computer Science and Software Engineering, May, Vol. 5, No. 5, pp.310-316.

Lee, H. and Jeon, D. (2015) 'A mobile ad-hoc network multi-path routing protocol based on biological attractor selection for disaster recovery communication', ICT Express, Vol. 1, No. 2, pp.86-89, Elsevier.

Patel, K.N. and Jhaveri, R.H. (2015) 'Isolating packet dropping misbehavior in VANET using ant colony optimization', International Journal of Computer Applications, Vol. 120, No. 24, pp.5-9.

Shoaib, M. and Song, W-C. (2013) 'Traffic aware optimized zone based hierarchal link state routing protocol for VANET', 24th International Symposium on Personal, Indoor and Mobile Radio Communications: Mobile and Wireless Networks, IEEE, pp.3117-3122, ISSN: 21669570.

Toutouh, J. et al. (2011) 'Performance analysis of optimized VANET protocols in real world tests', 7th IEEE International Wireless Communications and Mobile Computing Conference, pp.1244-1249.

Wang, T. and Wang, G. (2010) 'TIBCRPH: traffic infrastructure based cluster routing protocol with handoff in VANET', in Proceedings of 19th Annual Wireless and Optical Communications Conference (WOCC), IEEE, pp.1-5.

Wu, C., Ohzahata, S. and Kato, T. (2013) 'Flexible, portable, and practicable solution for routing in VANETs: a fuzzy constraint Q-learning approach', IEEE Transactions on Vehicular Technology, November, Vol. 62, No. 9, pp.4251-4263.

Wu, C., Ohzahata, S. and Kato, T. (2012) 'Fuzzy logic for multi-hop broadcast in vehicular ad hoc networks', A Chapter in a book Fuzzy Logic Emerging Technologies and Applications, InTechOpen, DOI: 10.5772/36768.

Wu, C., Yoshinaga, T. and Ji, Y. (2016) 'Context-aware unified routing for VANETs based on virtual clustering', IEEE 27th Annual International Symposium on Personal, Indoor, and Mobile Radio Communications (PIMRC).

Yuan, X., Elhoseny, M., El-Minir, H.K. and Riad, A.M. (2016) 'A genetic algorithm-based, dynamic clustering method towards improved WSN longevity', J. Netw. Syst. Manage., April, pp.21-46, Springer, DOI: 10.1007/s10922-016-9379-7. 